case report

\title{
Adult obstructing ileocolic intussusception
}

\author{
Ivan Žokalj ${ }^{1}$, Zvonimir Magaš ${ }^{2}$, Zlatko Pavčec ${ }^{1}$, Hussein Saghir ${ }^{1}$, Andrej Pal ${ }^{1}$, \\ Zvonimir Kolaric $^{3}$, Miljenko Marotti ${ }^{4}$
}

\author{
${ }^{1}$ Department of Radiology and Ultrasound, County Hospital Čakovec, Croatia; \\ ${ }^{2}$ Department of Surgery, County Hospital Čakovec, Croatia; ${ }^{3}$ Department of Internal Medicine, \\ County Hospital Cakovec, Croatia; ${ }^{4}$ Department of Diagnostic and Interventional Radiology, \\ University Hospital "Sisters of Mercy", Zagreb, Croatia
}

Background. We report a case of the adult obstructing ileocecal intussusception caused by carcinoma of the coecum.

Case report. A 44-year-old male patient has been admitted to the hospital with strong pain in the upper abdomen, vomiting and high amylase level in the blood serum $154 \mathrm{U} / \mathrm{L}$ (norm. 23-91 U/L at 37 ${ }^{\circ} \mathrm{C}$ ) and in the urine $792 \mathrm{U} / \mathrm{L}\left(0-400 \mathrm{U} / \mathrm{L}\right.$ at $\left.37^{\circ} \mathrm{C}\right)$. Sudden worsening of the patient's general condition on the $9^{\text {th }}$ day after the admission associated with clinical and radiological signs of bowel obstruction were the reasons to perform emergency computerized tomography (CT) after days of clinical observation and follow-up with abdominal X-rays and ultrasound (US). CT revealed multiple concentric rings with centrally placed soft-tissue structure with higher attenuation on post-contrast scans, "target mass". Right haemicolectomy with terminolateral ileotransversoanastomosis without preoperative reduction was performed. Intraoperatively aboral loops of the ileum were found prolapsed into the coecum and ascendant colon with carcinoma of the coecum (Dukes B, Astler-Coller B2) acting as a neoplastic lead point for intussusception.

Conclusions. The adult intussusception may be a rare cause of abdominal pain but it must be on the differential diagnosis list in the case of intermittent abdominal pain, especially with clinical and radiological signs of the bowel obstruction. The reported case supports the opinion that CT is the imaging method of choice for the adult intussusception.

Key words: intestinal obstruction; intussusception; adult; ileocecal valve

\section{Introduction}

Intussusception is a prolapse of a bowel loop with its mesenteric fold (intussuscep-

Received 18 September 2007

Accepted 30 September 2007

Correspondence to: Ivan Žokalj, MD, Department of Radiology and Ultrasound, County Hospital Čakovec, Croatia; E-mail: ivan.zokalj@ck.htnet.hr tum) into the lumen of a contiguous segment (intussuscipiens). ${ }^{1}$ Intussusception in adults is rare, it counts for $5-16 \%$ of all cases of all intussusceptions This condition is often at the end of the differential diagnosis list. ${ }^{2,3}$ The clinical presentation of intussusception in adults is variable in appearance. The signs and symptoms of the bowel obstruction predominate in 
$82 \%$ of cases. ${ }^{4}$ Patients usually complain on chronic intermittent abdominal pain or prolonged abdominal pain, nausea, vomiting and constipation. ${ }^{3,4}$ The most common reason for the operative intervention is the acute bowel obstruction. Unfortunately, the preoperative diagnosis of intussusception in adults is relatively rarely established. ${ }^{4}$ In most of the adult intussusception cases, in over $90 \%$, there is a pathological lead point. ${ }^{5}$ Malignant tumour is the lead point in approximately two thirds of colonic intussusceptions, but only in one quarter of enteric intussusceptions in adults. ${ }^{4}$

Abdominal computerized tomography (CT) is the most useful imaging diagnostic method for the diagnosis of intussusception. Ultrasound (US) is the second most effective imaging tool. ${ }^{6,7}$ Adult intussusceptions are usually treated surgically, especially colonic variants. Resection without reduction was suggested as the best method. Opinions differ among surgeons what is the best modality of the treatment of adult intussusceptions today. The role of preoperative reduction of adult intussusception is in the focus of the debate. ${ }^{7,8}$

\section{Case report}

A 44-year-old male patient had medical history of uretherolithiasis and chronic gastritis which has been treated with $\mathrm{H} 2$ antagonists. Two days before the admission he complained on upper abdominal pain and vomiting, which relieved spontaneously. On the day of admission, he complained again on upper abdominal pain. During the physical examination painless palpable mass which extended from epigastrum to the umbilicus with dimensions of $10 \times 5 \mathrm{~cm}$, has been found. First laboratory tests showed higher amylase level in the blood serum 154U/L (norm. 23-91 U/L at $\left.37^{\circ} \mathrm{C}\right)$ and the urine $792 \mathrm{U} / \mathrm{L}(0-400 \mathrm{U} / \mathrm{L}$ at $37 \mathrm{C}$ ) and normal results of WBC $9.1 \times$ $10^{9}$, erythrocytes $4.8 \times 10^{12}$, erythrocyte sedimentation rate $4 / 10$, C-reactive protein (CRP) $1.1 \mathrm{mg} / \mathrm{L}$.

Abdominal X-rays depicted bowel meteorism without distension in the ileocecal region and lienal flexure. The endoscopic evaluation of the upper gastrointestinal tract performed on the $4^{\text {th }}$ day after the admission revealed small oesophageal varices placed in the aboral third of the oesophagus just beneath cardia and a mild form of antral gastritis. The first abdominal US performed on the $5^{\text {th }}$ day after the admission did not show any abnormalities. The control abdominal US on the $7^{\text {th }}$ day after the admission depicted expansive formation in the epigastrium with concentrically placed hypoechoic and hyperechoic layers with a maximal diameter of $10 \mathrm{~cm}$ ("target mass"). The working diagnosis was acute pancreatitis with suspected pseudocyst of the pancreas.

In the following days the patient developed clinical signs of bowel obstruction. On the $9^{\text {th }}$ day after the admission the patient had severe and much stronger pain in the upper abdomen in comparison with the first day of this episode. Control abdominal X-rays showed the distension of enteric loops without earlier depicted colonic meteorism. The follow-up abdominal US, performed on the same day, revealed earlier depicted "target mass" in the upper abdomen with bigger maximal diameter of $12 \mathrm{~cm}$ and more clearly delineated contours from the surrounding structures. The patient underwent abdominal CT which revealed multiple concentric rings on the place of sonographically depicted "target mass" with centrally placed soft-tissue structure which had higher attenuation on post-contrast scans. Control laboratory tests revealed again normal levels of WBC, erythrocytes, CRP and decreased amylase blood serum (111 U/L) and urine level (1041 U/L). 


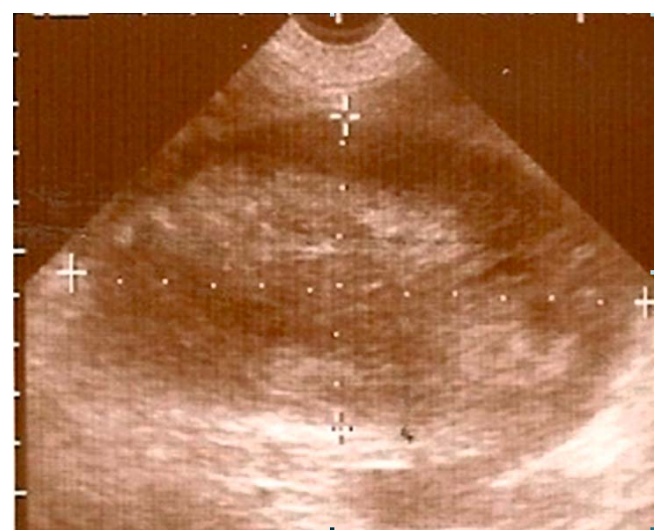

Figure 1. Abdominal ultrasound - axial plane. Expansive formation in the epigastrium with concentrically placed hyper and hypoechoic layers - "target mass".

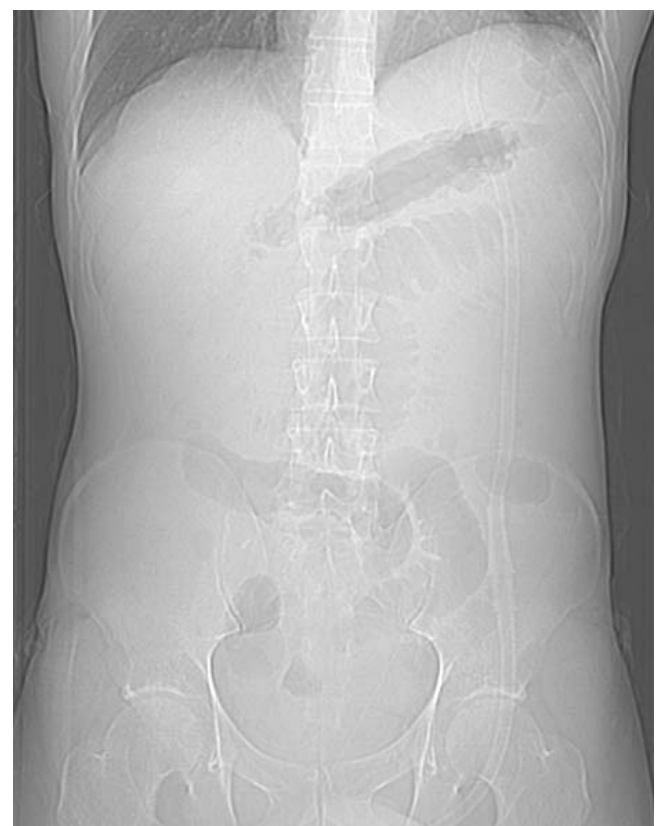

Figure 2. AP scout view of abdominal CT - coronal plane. Distension of the small bowel loops - bowel obstruction.

Due to a worsen clinical condition of the patient the emergency right haemicolectomy with terminolateral ileotransversoanastomosis was performed. Ileocolic invagination with terminal ileum invaginated in transverse colon was found. The polypoid

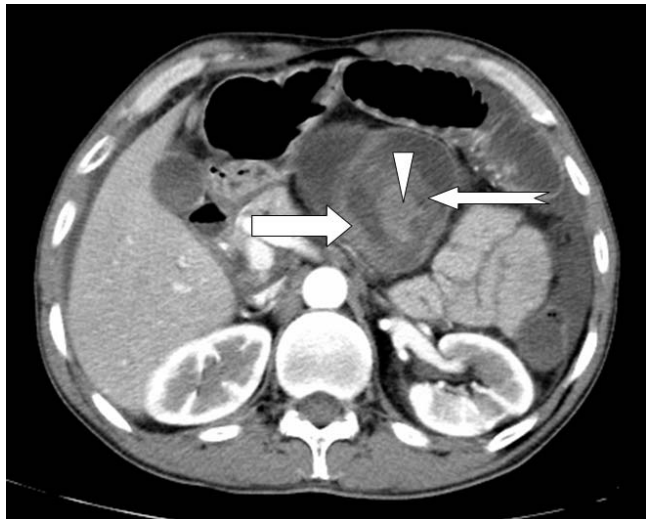

Figure 3. CT of the abdomen - post-contrast axial scan - horizontal plane. Mass consisted of multiple concentric rings placed in the middle upper abdomen with external hyperdensity ring (returning intussusceptum and intussuscipiens - white right arrow ), hypodensity ring, internal hiperdensity ring (canal and wall of intussusception - white notched right arrow) and soft tissue density structure in a central position - a malignant lead point (white arrowhead). Dilatation of small bowel loops.

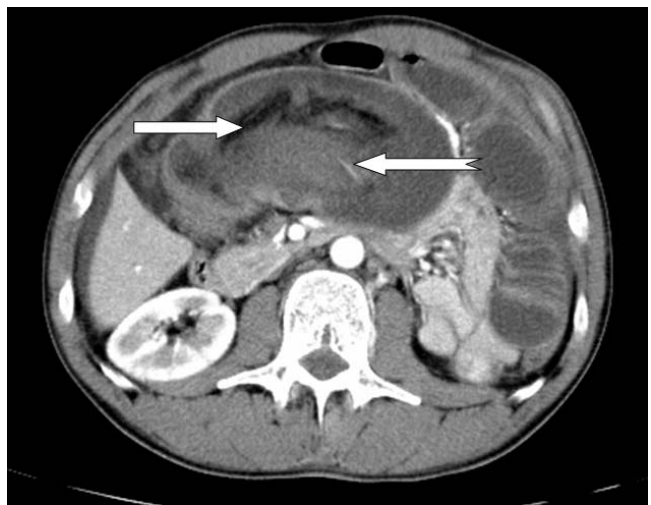

Figure 4. Abdominal CT post-contrast axial scan - horizontal plane. Crescent-shaped fat density due to the eccentrically placed mesentery (white right arrow). Mesenterial vascular structures (white notched right arrow).

form of coecal carcinoma served as a lead point in this case of the adult intussusception. The result of the patohystologic analysis was adenocarcinoma of the coecum, classified as Dukes B stage, Astler-Coller B2 and T3NOMX. The patient was discharged 
from the intensive care unit on the second day after the surgical intervention. The patient's general condition was improved and he was discharged from the hospital after the total stay of 21 days.

\section{Discussion}

The case presented in this article represents difficulties in establishing the aetiology of the prolonged upper abdominal pain connected with non-specific clinical signs and laboratory test results. The diagnosis of the intussusception in adults is rarely established preoperatively. The diagnosis is usually established during the surgery performed because of the bowel obstruction. The adult intussusception is the cause of bowel obstruction in only $1 \%$ of patients. ${ }^{7}$ Cross-sectional imaging tools like US and $\mathrm{CT}$, especially modern fast scanners with possibilities of volumetric scanning, make the preoperative diagnosis of the adult intussusception more possible.

Intussusceptions are usually divided according to the location. Dean, Ellis and Sauer have classified intusussceptions into four groups:

1 Enteric-small bowel invagination into small bowel

2 Ileocecal-the ileocecal valve being the leading point of the intususception

3 Ileocolic-ileum protruding through the ileocecal valve with the coecum remaining stationary

4 Colocolic-colon invaginating into colon. ${ }^{4}$ Organic lesions are the underlying pathologic cause in $10-20 \%$ of the patients. Aetiology of intussusception in adults is demonstrated in $70 \%$ to $90 \%$. Large bowel intussusceptions are usually caused by malignant tumours. In small bowel intussusceptions a lead points are predominantly non-malignant tumours, like lipomas and polyps. Enteroenteric intussusceptions, in the transient non-obstructing form, can be found in patients with coeliac disease and Crohn's disease where a pathologically changed bowel wall with altered peristalsis can form a leading edge for the intususceptum. $1,3,4,9,10$

In the study published in 1999, conducted by Warshauer and Lee, less than $1 / 3$ of adult intussusceptions depicted with CT or MR had a neoplastic lead point. Non-neoplastic intussusceptions were mostly enteroenteric, significantly shorter and smaller in diameter. ${ }^{3}$

The bowel obstruction is uncommon in adult non-neoplastic intussusceptions. ${ }^{3}$ Non-neoplastic adult intussusceptions can be transient and may not demand aggressive work-up. ${ }^{1,3}$

Diagnostic studies in evaluation of the suspected intusussception are plain abdominal film (abdominal X-rays), US, barium enema (irigography), CT, magnetic resonance (MR) and colonoscopy. ${ }^{3,4,6}$

$\mathrm{CT}$ is the diagnostic method of choice because of its non-invasiveness and short scanning time on modern scanners. CT can also depict changes which are responsible in development of intussusception (the leading edge).

CT appearance of intussusception has a few different forms:

1 Target pattern - multiple concentric rings

2 Reniform pattern - renal like or bilobed mass with peripheral high attenuation values

3 Sausage-shaped pattern - sausage-shaped mass with alternating areas of low and high attenuation. ${ }^{1,11}$

On axial CT scans a target lesion is often depicted (multiple concentric rings) with alternating circumferential areas of high and low attenuation. The external ring on axial scans (or outer cylinder) usually consists of returning intussusceptum and intussuscipiens. The crescent of the mesenteric fat forms the middle ring (middle cylinder) and 
the internal ring (central cylinder) contains the wall of intussusception and canal. ${ }^{1}$

Using the alternative cross-sectional methods, like US and MR, the intussusception will also be depicted as a lesion with the target appearance (multiple concentric rings). In the early phase the intussuception is usually a "target mass" associated with the bowel obstruction. The progression of the disease causes the bowel wall thickening and layering on the images. Finally, if the patient is not treated, the bowel wall necrosis appears as an amorphous mass on the images. ${ }^{1,11}$ In this case we had a target pattern of an ileocolic intussusception on US and CT images.

Non-specific clinical signs and symptoms were the main reasons why CT was not used earlier for the evaluation of this patient. Abdominal CT has been originally planned because the treating physician assumed that the main cause of the upper abdominal pain in this case is the acute pancreatitis complicated with pseudocyst of the pancreas. Sudden worsening of the patient's general condition connected with clinical and radiological signs of the bowel obstruction were the reasons to perform emergency CT which depicted the ileocecal intussusception with a characteristic target pattern. In this case, CT results gave the key reason to surgeons for their decision to perform an emergency surgical treatment.

The form of the surgical treatment depends on the patient's medical history and intraoperative findings. Opinions about the treatment of different variants of adult intussusception differ today, especially colonic intussception should be reduced before the resection. ${ }^{6,7,8}$ The treatment in the adults for colonic variants of intussception is always operative and the resection without reduction is recommended according to Azar and Berger. ${ }^{7}$ Sarr in his invited commentary to article of Omori et al. published in 2003 proposed the preoperative reduction in selective cases of intussusceptions to allow an elective procedure. ${ }^{8}$ The preoperative reduction of the colonic intussusception may help to reduce the extent of the surgical procedure, for example, to treat the ileocolonic intussusception with a small bowel resection instead of a right hemicolectomy, according to Sarr. ${ }^{8}$ Benign enteric lesions without adhesions and benign colonic lesions are usually treated with the resection to prevent a recurrent intussusception. ${ }^{7}$

\section{Conclusions}

In this case we report about a patient with non-specific clinical symptoms characterized with the intermittent abdominal pain and with the slowly progressive bowel obstruction. After nine days of clinical observations and a follow-up with abdominal X-rays and US the upper abdomen lesion with target appearance was detected. Because of the worsened general condition the patient underwent the CT examination which demonstrated typical findings of the intussusception. CT findings and a worsened clinical picture were the reasons for an urgent surgical intervention. The right haemicolectomy without a preoperative reduction of the intussusception has been done. The adult intussusception may be a rare cause of the abdominal pain but it must be on the differential diagnosis list in the case of the intermittent abdominal pain, especially with clinical signs of the bowel obstruction. The reported case also supports the opinion that CT is the imaging method of choice for the adult intussusception. 


\section{References}

1. Catalano O. Transient small bowel intussception: CT findings in adults. Br J Radiol 1997; 70: 805-8.

2. Agha FP. Intussusception in adults. AJR Am J Roentgenol 1986; 146: 527-31.

3. Warshauer DM, Lee JKT. Adult intussusception detected at $\mathrm{CT}$ or MR imaging:Clinical-imaging correlation. Radiology 1999; 212: 853-60.

4. Stubenbord WT, Thorbjarnarson B. Intussusception in adults. Ann Surg 1970; 172: 306-10.

5. Garner JP, Haldipur, Ravi K, Amarnath TS, Gupta $\mathrm{R}$. Colonic intussusceptions in adults: three cases and review of the literature. Indian J Surg 2006; 68: 322-4.

6. Omori $\mathrm{H}$, Asahi $\mathrm{H}$, Inoue $\mathrm{Y}$, Irinoda $\mathrm{T}$, Takahashi M, Saito K. Intussusception in adults: a 21-year experience in the University-affiliated Emergency Center and indication for nonoperative reduction. Dig Surg 2003; 20: 433-9.

7. Azar T, Berger DL. Adult intussusception. Ann Surg 1997; 226: 134-8.

8. Sarr M. Commentary on Intussusception in adults: a 21-year experience in the University-affiliated Emergency Center and indication for nonoperative reduction. Dig Surg 2003; 20: 438-9.

9. Merine DS, Fishman EK, Jones B, Siegelman SS. Enteroenteric intussusception: CT findings in nine patients. AJR Am J Roentgenol 1987; 148: 1129-32.

10. Knowles MC, Fishman EK, Kuhlman JE, Bayless TM. Transient intussusception in Crohn disease: CT evaluation. Radiology 1989; 170: 814.

11. Eisen LK, Cunningham JD, Aufses AH Jr. Intussusception in adults: institutional review. $J$ Am Coll Surg 1999; 188: 390-5. 